\section{Ambulant erworbene Pneumonie: Diagnose nicht immer korrekt}

Daniel $\mathrm{P}$ et al. Adults miscoded and misdiagnosed as having pneumonia: results from the British Thoracic Society pneumonia audit. Thorax 2017; DOI: 10.1136/thoraxjnl-2016-209405

Diagnose-Codes werden zunehmend genutzt, um bei Pneumonien die Behandlungsergebnisse verschiedener Institutionen zu vergleichen. Allerdings unterliegt die Codier-Praxis größeren Schwankungen, was gerade bei Pneumonien an gelegentlichen Schwierigkeiten liegt, eine definitive Diagnose zu stellen, wenn beispielsweise keine Röntgenmöglichkeit vorhanden ist. Die Britische Thorax-Gesellschaft betrachtete nun fehlcodierte Patienten einmal genauer.

Für das Audit lud die Gesellschaft Institutionen des National Health Service (NHS) zur Teilnahme ein. Diese sollten erwachsene Patienten identifizieren, die zwischen Dezember 2014 und Januar 2015 eine ICD10 Entlassdiagnose mit den Schlüsseln J12-J18 hatten. Die Autoren überprüften anschließend die Einträge dieser Patienten in den Krankenakten und ordneten diese einer Gruppe mit ambulant erworbener Pneumonie oder einer Gruppe ohne Pneumonie zu. Kriterien für die Zuordnung zur PneumonieGruppe waren ein Alter von mindestens 16 Jahren, ein neu aufgetretenes Infiltrat auf einer Röntgenaufnahme des Thorax, Zeichen und Symptome einer Infektion der unteren Atemwege sowie keine Krankenhausentlassung in den vorausgehenden 10 Tagen und keine Immunsuppression. Anschließend verglichen die Autoren korrekt und unkorrekt codierte Patienten miteinander.

In die Analyse gingen 6660 Patienten in der Gruppe mit korrekt codierter Pneumonie ein sowie 1251 Patienten mit unkorrekt codierter Pneumonie. Nahezu alle Patienten (95\%) mit einer Fehlcodierung erhielten bei Aufnahme eine antibiotische Behandlung; Symptome, die mit einer Infektion der unteren Atemwege kompatibel waren, lagen bei $74,6 \%$ dieser Patienten ( $n=933$ ) vor, die Rönt- genaufnahme des Thorax zeigte bei $38,9 \%$ von ihnen $(n=487)$ einen Normalbefund. Fehlcodierte Patienten waren im Median älter als die korrekt codierten (80 vs. 78 Jahre) und hatten zu einem höheren Anteil ernste Begleiterkrankungen (0-1 Komorbiditäten $61,3 \%$ vs. $68,9 \%$, $2-4$ Komorbiditäten $38,0 \%$ vs. $30,6 \%$ und $\geq 5$ Komorbiditäten $0,7 \%$ vs. $0,5 \%$ ). Darüber hinaus zeigten unkorrekt codierte Patienten weniger Symptome einer Atemwegsinfektion sowie eine niedrigere 30 -Tages-Mortalität mit $14,3 \%$ vs. 17,0\% (angepasste Odds Ratio 0,75).

FAZIT

Nach Ansicht der Autoren sind Maßnahmen nötig, um das Ausmaß von Fehlcodierungen und Fehldiagnosen zu ermitteln und zu reduzieren, gerade wenn solche Datensätze genutzt werden sollen, um Qualitätsverbesserungen bei der Pneumonie-Behandlung zu beurteilen. Auf klinischer Ebene würde eine zuverlässigere Unterscheidung von Patienten mit und ohne Pneumonie zielgerichtetere Therapiestrategien erlauben und den ungerechtfertigten Einsatz von Antibiotika reduzieren.

Dr. med. Johannes Weiß, Bad Kissingen 